\title{
A Novel Conductor-Backed Coplanar-Waveguide Coupled Structure
}

\author{
Chun-Lin Jiao and Chun Hsiung Chen \\ Department of Electrical Engineering, \\ National Taiwan University, Taipei 10617, Taiwan
}

\begin{abstract}
A new coplanar-waveguide coupled structure is proposed by incorporating a backed conductor on the other side of the substrate. For this new coupled structure, the effective dielectric constants as woll as the characteristic impedances of the odd and even modes are calculated by the spectral-domain method. In this study, the proposed coupled structure is characterized by carefully examining the t'elated coupling coefficient and the associated field distributions.
\end{abstract}

\section{INTRODUCTION}

The coplanar waveguides (CPW) receive much attention recontly due to the merits of easy in connecting shunt and series components. One disadvantage of conductor-backed coplanar waveguides is the unwanted leakage phenomenon associated with the parallel-plate transmission line structure. In this study, the coupling effect due to finitc-width backed conductor is utilized to transfer the power between two coplanar waveguides so that a now corpled structure may be implemented.

Various coplanar-wavogicie coupled structures have been proposed. The broadsidecoupled coplanar-waveguide structures have the merits of tight coupling and high effective dielectric constant ratio [1] [2], but these structures are only compatible with multilayer MMIC technology. Édge-coupled structures could not achieve tight coupling as the pre-

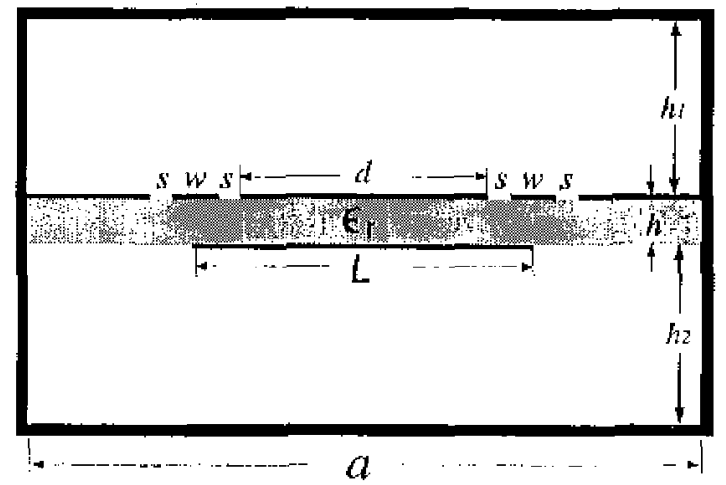

Fig. 1. Cross section of conductor-buckod coplanar-waveguido coupled structuro. $(w=$ $1 \mathrm{~mm}, s=0.5 \mathrm{~mm}, c_{r}=2.5, h=0.635 \mathrm{~mm}$, $\left.h_{1}=h_{2}=60 \mathrm{~mm}, a=1000 \mathrm{~mm}\right)$.

vious ones but the whole structures are or the same plane $[3]-[4]$. In this study, a new coupled structure which makes use of a backed conductor to couple two coplanar waveguides on the same side of the substrate is proposed and examined.

\section{FORMULATION}

Consider a symmetric conductor-backed coplanar-waveguide compled structure as shown in lig. 1 in which a backed conductor of width $L$ is introduced in the other substrate side of two coplanar. waveguiclos. 'Two coplanar waveguides have iclentical strip width $w$ and slot width s, and tho dielectric constant and thickness of tho substrate are $\epsilon_{\text {r }}$ and $h$, re 


\section{odd CPW mode:}

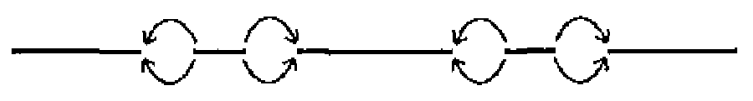

even CPW mode:

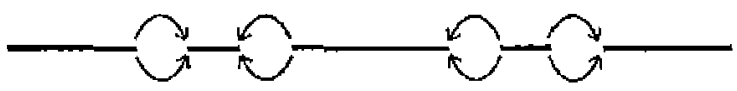

Pig. 2. Eloctric field distributions for odd and even CPW modes.

spectively. The coupling mechanism is mainly ontrolled by the width $L$ of backed conductor (Fig, 1). 'To simplify the analysis, our coplanar-wavegtide couplod structure is enclosed by a conducting box.

Although many futidamental modes may be supported by the multi-conductor system (Fig. 1), we aro mainly concerned with the odd and even CPW modes with field distributions as shown in lig. 2. These two modes are responsiblo for the coupling mechanism associated with two coplanar wavoguides. By using the spectral-donain method, the offective dielectric constants $\epsilon_{e j f}$ as well as characteristic impedances $Z_{0}$ of odd and cven C]W modes are calculated, from which the coupling characteristics of the structure may be examined.

\section{NUMERICAL RESULTS}

Fig. 3 shows the effective lielectric constants and characterstic impedances of old and even CPW modes, is well as the rolated coupling coefficient $C$ under the condition of $L=0$. Without backed conductor on the other side of the substrate, the effective dielectric constants of the two modes are almost identical as $d>5 \mathrm{~mm}$, and the coupling coefficient is smaller thin -50dls when $d>5 \mathrm{~mm}$, showing that the courling between two coplenar wave-

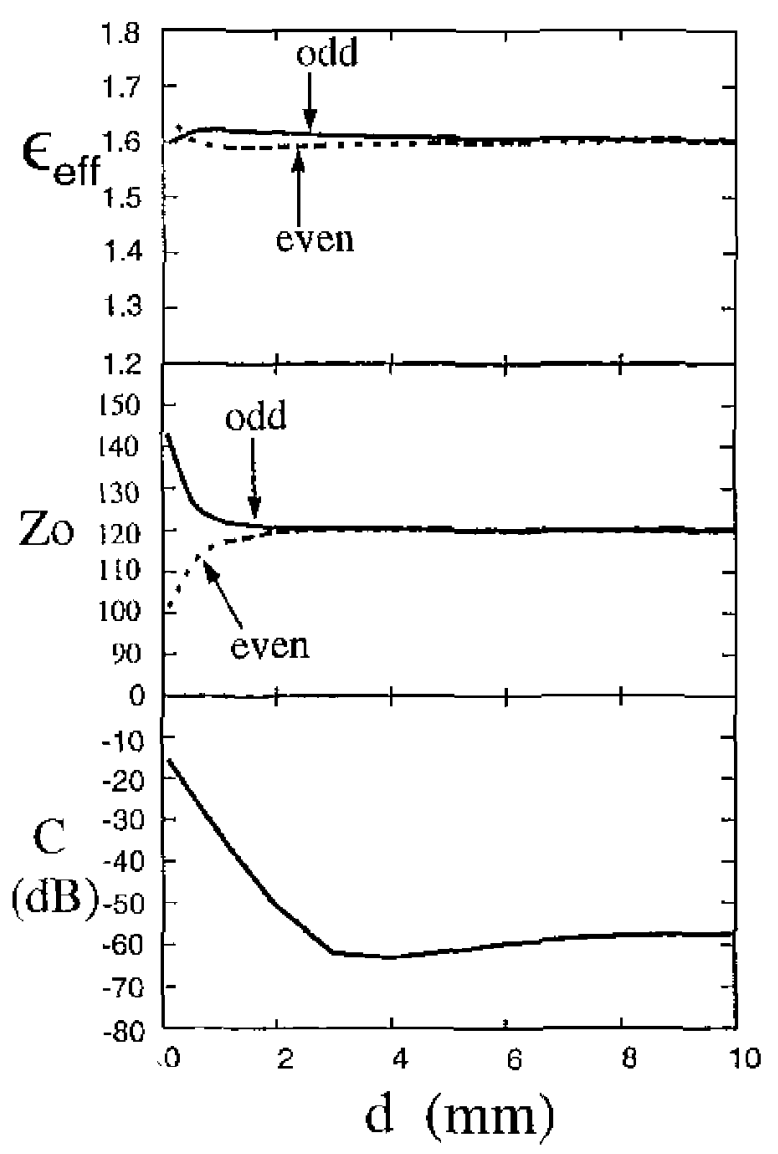

Tig. 3. Liffective diclectric constants, chatacteristic impedances of odd and even modes, and related coupling coefficiont $(L=0)$.

guides is not significant.

For the case with $d=5 \mathrm{~mm}$, and the width $L$ of backed conductor varying from $2 \mathrm{~mm}$ to $12 m m$, the resultis for $\epsilon_{c f f}, Z_{0}$ and $C$ are shown in Fig. 4. For $L>5 \mathrm{~mm}$, the backed conductor under the substrate is wide enough to cover the two transmission lines, and this makes the difference between the two $c_{e f f}$ become obvious. With obvious difference between the characteristic impedances $Z_{0}$, the coupling coefficient reaches $-12 \mathrm{~dB}$ as $L>9 \mathrm{~mm}$, much linger than the case of $I_{i}=0$. For this case of $I$. 9mm, the two conductors of widths $d$ and $L$ may be regarded as a paraltel-plate transmission line and the coupling elfoct assoclated with the TEM wave bocomes significant. 


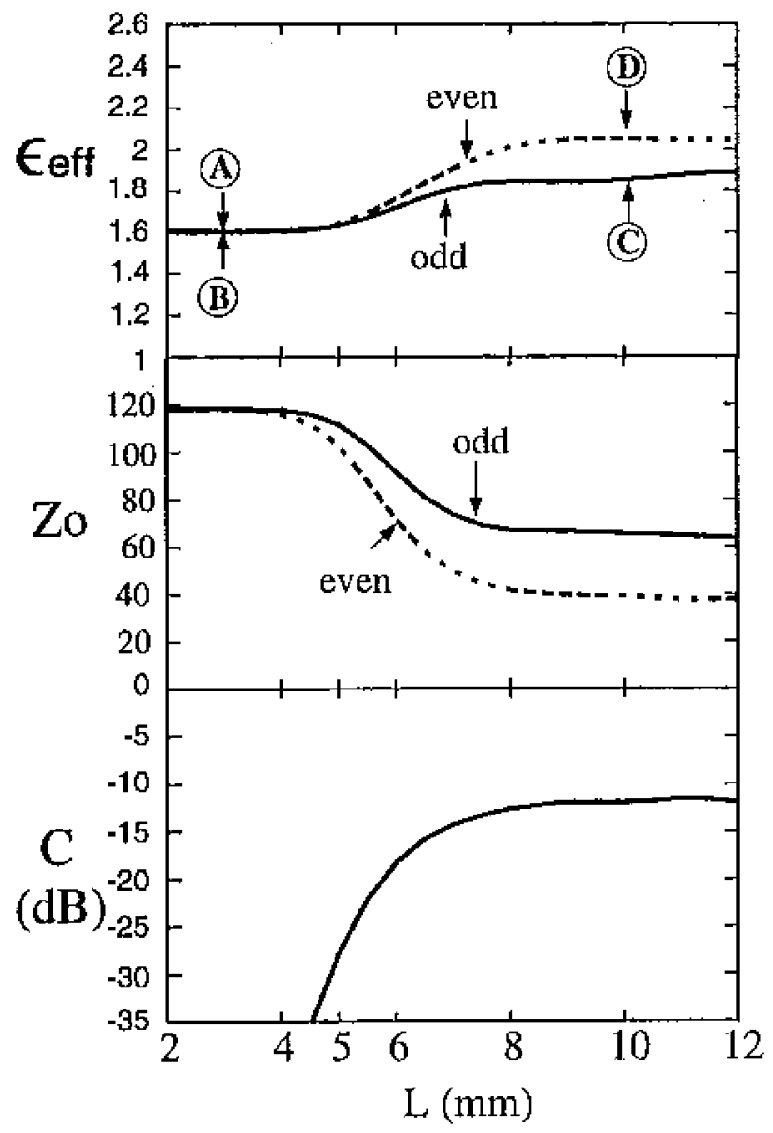

Fig. 4. Wffective dielectric constants, charactcristic impedances of odd and even modes, and related coupling coefliciont $(d=5 \mathrm{~mm})$.

This effect makes the power transfor from one coplanar waveguide to the other easily even in the lower frequency range.

Fig. 5 shows the elcctric field distributions corresponding to the cases $\mathrm{A}, \mathrm{B}, \mathrm{C}, \mathrm{D}$ of $\mathrm{Fig}$. 4. 'I'he cases $A$ and $B$ are for the old and even CPW modes with $L=3 \mathrm{~mm}$. Since the width $L$ of backed-conductor is narrower than the line spacing $(d=5 \mathrm{~mm}$ ), the coupling of fect is weak and the electric ficlds between two central conductors of widths $d$ and $L$ are very small. For the case with $L=10 \mathrm{~mm}$, the electric fields for odd and even CPW modes are shown as $\mathrm{C}$ and $\mathrm{D}$ in Fig. 5. The coupling offect between two coplanar waveguides is more obvious than the previous one and the fields for odd CPW mode between the central conductors are stronger than those for $L=3 \mathrm{~mm}$, a consequence of the excited TEM wave in the parallel-plate structine.

\section{CONCLUSIONS}

In this work, the coupling offect due to backed conductor is utilized to implement a new coupled structure coruposed of two coplanar waveguides. Without backed conductor on the other side of the substrate, the coupling phenomenon is not obvious. The proposed coupled structure shows significant coupling when the backed conductor width $L$ is wider enough to cover the two coplanar waveguides.

\section{REFERENCES}

[1] C. Nguyen, "Broadside-coupled coplanar waveguides and thoir ond-coupled bandpass filter applications," ILLE Trans. $\mathrm{Mi}$ crowave Theory Tech., vol. 40, pp. 2181\% 2189, Dec. 1992.

[2] I. P. Hong, Y. K. Loe and H. K. Park, "Dispersion clatacieristics of a broadsidecompled coplanar waveguide," Dectronics Letters, vol.33, pp. 965-966, May 1997.

[3] P. Singkornrat and J. A. ]3uck, "Picosecond pulse propagation in coplanar waveguide forward directional couplers ," WLEL Irans. Microwave Theory Tech, vol. 39, pp. 1.025-1028, June 1991.

[4] G. Ghione and C. U. Naldi, "Coplanar waveguides for MMIC applications: effect of upper shielding, conductor backing, finite-extent ground planes, and lino-toline coupling," IEEE Trans. Microwave Theory Tech., vol. 35, pp-260. 267, March 1987. 

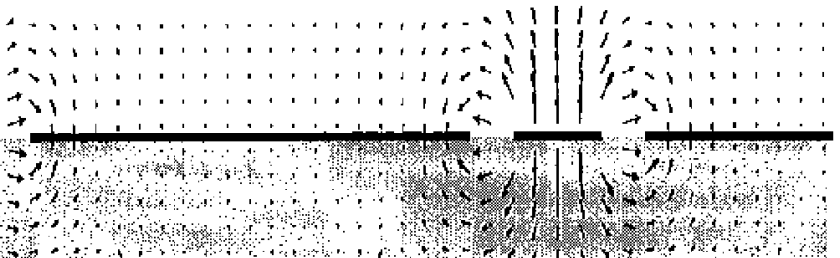

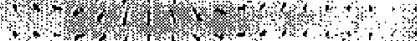

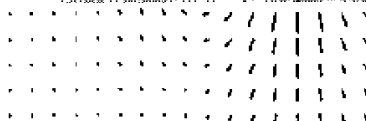

(B)

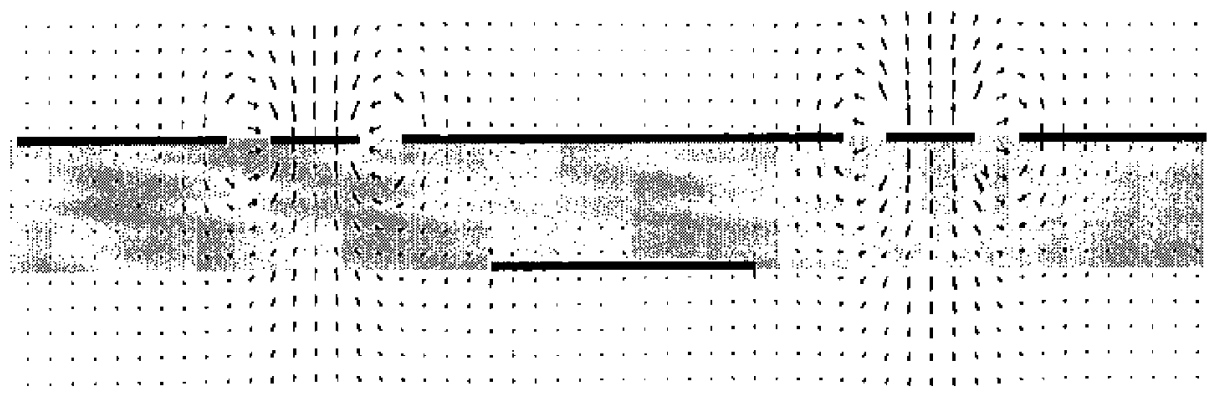

(C)

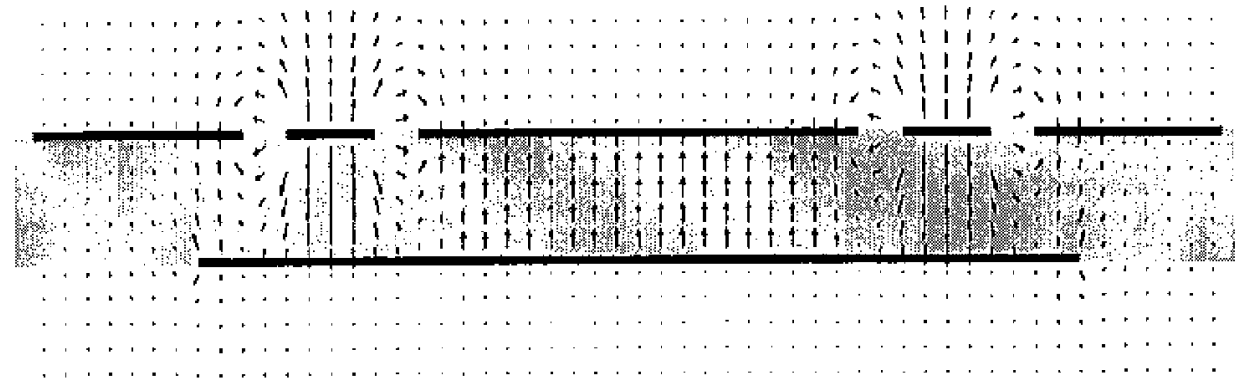

(D)

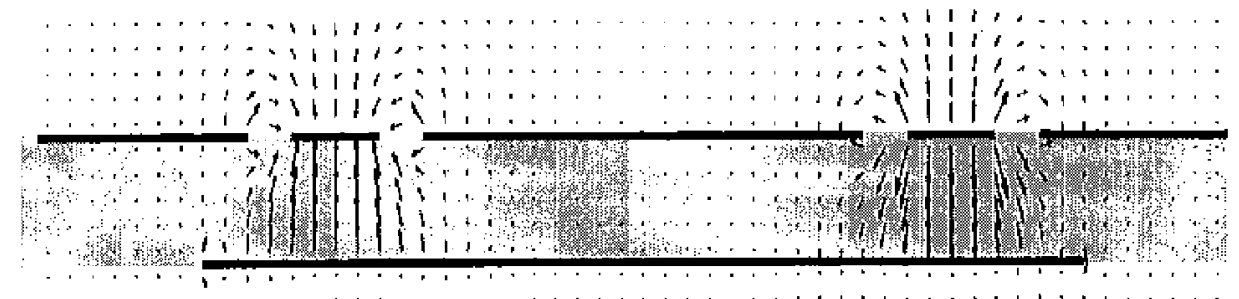

Fig. 5. likectric field distributions for $(\Lambda)$ odd CPW mode with $L=3 \mathrm{~mm}$, (B) even CPW mode witl $L=3 \mathrm{~mm}$, (C) odd CPW mode with $L=10 \mathrm{~mm}$, and (D) even CPW mode with $L=10 \mathrm{~mm}$. 